\title{
Monitoring the effect of heparin by measurement of activated clotting time during and after percutaneous transluminal coronary angioplasty
}

\author{
B Rath, D H Bennett
}

\begin{abstract}
The anticoagulant effect of heparin during percutaneous transluminal coronary angioplasty was monitored by measurements of the activated clotting time in two studies that compared the effects of a single bolus of heparin with those of a bolus of heparin combined with a continuous infusion of the drug. In a preliminary study 40 patients received a single heparin bolus of 10000 units (protocol I) and $a$ further 40 patients received both a heparin bolus of 10000 and a continuous infusion of heparin at a rate of 2000 units per hour (protocol II). During the first 45 minutes, nine patients $(23 \%)$ in protocol I but only two patients $(5 \%)$ in protocol II were found to be inadequately anticoagulated. For 24 hours after angioplasty both groups received an infusion of heparin at the rate of 2000 units per hour which led to consistent anticoagulation in $73(91 \%)$ of patients. In a subsequent randomised study, 40 patients received heparin according to either protocol I or II. Protocol II was again found to lead to a higher rate of adequate anticoagulation. During the first $60 \mathrm{~min}$ utes 11 patients $(55 \%)$ in protocol I but only three patients $(15 \%)$ in protocol II were inadequately anticoagulated. In addition, the activated clotting time of arterial blood in the first $\mathbf{3 0}$ minutes was significantly higher than that of venous blood in $70 \%$ of the patients.
\end{abstract}

$A$ bolus of heparin (10000 units) together with an infusion of 2000 units per hour should be routinely given during coronary angioplasty. The effects of heparin, which can vary considerably from patient to patient, should be monitored by the measurement of the activated clotting time of arterial blood.

\footnotetext{
Regional Cardiac Centre, Wythenshawe

Hospital, Manchester Manches B Rath
D H Bennett

Correspondence to Dr D H Bennett, Regional Cardiac Centre,

Wythenshawe Hospital Manchester M23 9L T.

Accepted for publication 10 May 1989
}

Heparin is administered routinely during percutaneous transluminal coronary angioplasty ${ }^{1}$ in order to prevent thrombus formation. Commonly, 10000 units of heparin is given at the start of percutaneous transluminal coronary angioplasty and a further 5000 units is administered if the procedure lasts for more than one hour. ${ }^{2}$ The effects of heparin, however, vary considerably from patient to patient $^{3}$ so that some may be at risk from inadequate anticoagulation.
The activated clotting time reflects heparin activity. ${ }^{4}$ Activated clotting time can be rapidly and repeatedly measured in the catheterisation laboratory by an automated instrument. The purpose of this study was to investigate the value of measurement of activated clotting time during percutaneous transluminal coronary angioplasty and to attempt to develop a regimen of heparin administration that would provide consistently effective anticoagulation during the procedure.

Some recommend routine infusion of heparin for 24 hours after the procedure to prevent thrombus formation at the site of dilatation. ${ }^{5}$ Others suggest that such infusion should be given only when the procedure is complicated by coronary artery dissection. ${ }^{6}$ Many of the complications of percutaneous transluminal coronary angioplasty, including restenosis, are related to intravascular thrombus formation. ${ }^{78} \mathrm{~A}$ subsidiary purpose of this study was to try to establish a suitable dosage regimen for continuous heparin infusion if indicated and also to see whether such a regimen had a pronounced effect on the rate of coronary restenosis.

\section{Patients and methods}

Two studies were carried out. In each study, patients received either a bolus injection of heparin or a bolus of heparin together with a continuous infusion.

\section{PRELIMINARY STUDY}

Observations were made in 80 consecutive patients undergoing percutaneous transluminal coronary angioplasty for stable or unstable angina (table).

The anticoagulant effect of heparin was monitored every 15 minutes during percutaneous transluminal coronary angioplasty by measurement of the activated clotting time by a

\section{Patients' details}

\begin{tabular}{lll}
\hline & Protocol I & Protocol II \\
\hline Number of patients & 60 & 60 \\
Age (yr) & $40-63$ & $43-68$ \\
Sex: & (mean 58) & (mean 54) \\
$\quad$ Male & 38 & \\
$\quad$ Female & 22 & 40 \\
Type of angina: & & 20 \\
Stable & 42 & 48 \\
Unstable & 18 & 12 \\
\hline
\end{tabular}


Hemochron blood coagulation timing system (International Technidyne Corporation, New Jersey, USA). Blood samples were collected from the angioplasty guiding catheter after aspiration of $6 \mathrm{ml}$ of blood. Whole blood $(2 \mathrm{ml})$ was placed in a Hemochron test tube containing $12 \mathrm{mg}$ of Celite and a cylindrical bar magnet. The test tube was shaken vigorously ten times and was placed in the instrument's incubated test well, which has a magnet detector at its base. The temperature was maintained at $37^{\circ} \mathrm{C}$ by the incubator. The cylindrical bar magnet remained close to the detector as the tube slowly rotated. When a fibrin mass formed, its adhesion to the magnet and a plastic structure within the tube caused the magnet to rotate with the tube. The magnet was thereby displaced from its initial position adjacent to the detector. This activated the timer and an audible tone signalled this end point. The coagulation time was displayed in seconds.

The effective anticoagulation was defined as an activated clotting time of $>250 \mathrm{~s}$.

The first 40 patients received a single bolus of 10000 units of heparin at the start of the procedure (protocol I). The bolus of heparin was administered through a sheath in the femoral artery which was then flushed with 10 $\mathrm{ml}$ of saline. The second group of 40 patients received 10000 units of heparin as a bolus injection immediately followed by continuous infusion of heparin 2000 units per hour (protocol II). If the activated clotting time fell below $250 \mathrm{~s}$ an additional bolus of 5000 units of heparin was given.

For 24 hours after percutaneous transluminal coronary angioplasty had been completed both groups received a continuous infusion at a rate of 2000 units per hour. Activated clotting time was measured at intervals over 24 hours on at least four occasions.

\section{RANDOMISED STUDY}

In a subsequent study, a further 40 patients (table) were randomised to receive heparin according to either protocol I or II. In this study, the route of heparin administration was randomised so that the drug was given through a sheath in either the femoral vein or in the femoral artery. The anticoagulant effect of heparin was monitored every 15 minutes for 60 minutes. Samples were collected, after aspiration of $6 \mathrm{ml}$ of blood, from both the guiding catheter in the aorta and the sheath in the femoral vein. Both the venous and arterial activated clotting time measurements were assessed simultaneously. A HemoTec (Colorado, USA) machine was used to facilitate simultaneous activated clotting time measurements of two blood samples.

Like the Hemochron machine, the HemoTec blood coagulation timing system also detected in vitro clot formation. One $\mathrm{ml}$ blood was collected in cartridges containing the reagent $(0.1 \mathrm{ml}$ of kaolin activator) and a plunger. The machine was operated by dropping the plunger through the test sample at programmed intervals. When clotting occurred, the clot retarded the fall of the plunger and this was detected photo-optically. An audible tone signalled the end point.

To assess the variability in activated clotting time measurements, duplicate samples were obtained five minutes apart from 10 other patients. The samples were analysed by a single operator (five patients) and two operators (five patients) using the Hemochron machine.

\section{Results}

\section{PRELIMINARY STUDY}

Activated clotting time values (protocol I and II) Of those patients receiving heparin according to protocol I, six, two, and one patients were inadequately anticoagulated at 15,30 , and 45 minutes, respectively. Whereas only two patients were inadequately anticoagulated by protocol I (at 30 and 45 minutes). Thus nine $(23 \%)$ patients in protocol $\mathrm{I}$ and two $(5 \%)$ patients in protocol II were inadequately anticoagulated and required further doses of heparin.

Activated clotting time values during continuous heparin infusion after angioplasty

During the continuous intravenous heparin infusion after completion of percutaneous transluminal coronary angioplasty, most patients achieved a steady activated clotting time value; however, seven $(9 \%)$ patients were inadequately anticoagulated. Their heparin dosage was not altered. The incidence of restenosis in those who were adequately anticoagulated was $28 \%$, similar to our previous 80 consecutive patients undergoing angioplasty and to restenosis rates reported in many other reports.

\section{RANDOMISED STUDY}

Activated clotting time values (protocol I and II) Figures 1 and 2 show the activated clotting time values in arterial blood of patients receiving heparin according to protocols I and II. In patients receiving heparin according to protocol I, three, one, two, and five patients were inadequately anticoagulated at $15,30,45$, and 60 minutes, respectively. Whereas in

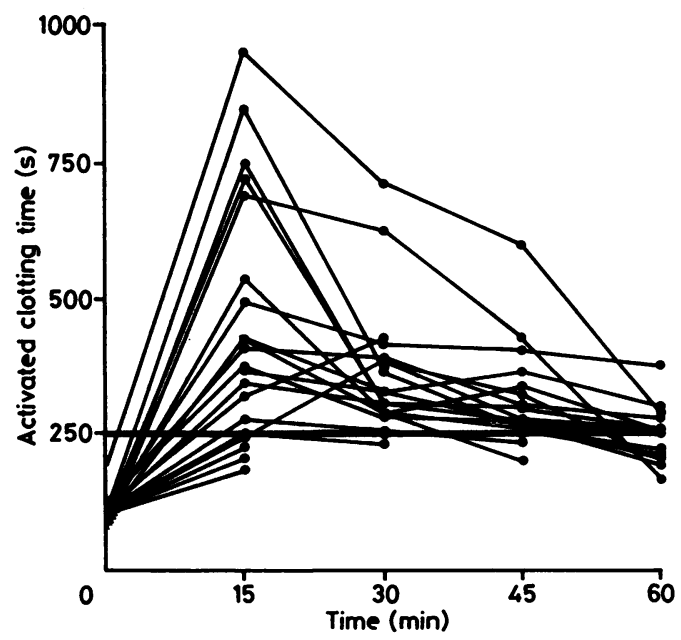

Figure 1 Activated clotting time in patients receiving a single bolus of 10000 units of heparin at the start of the procedure (randomised study). 
Figure 2 Activated clotting time in patients heparin as a bolus followed by continuous infusion of 2000 units per hour (randomised study). receiving 10000 units

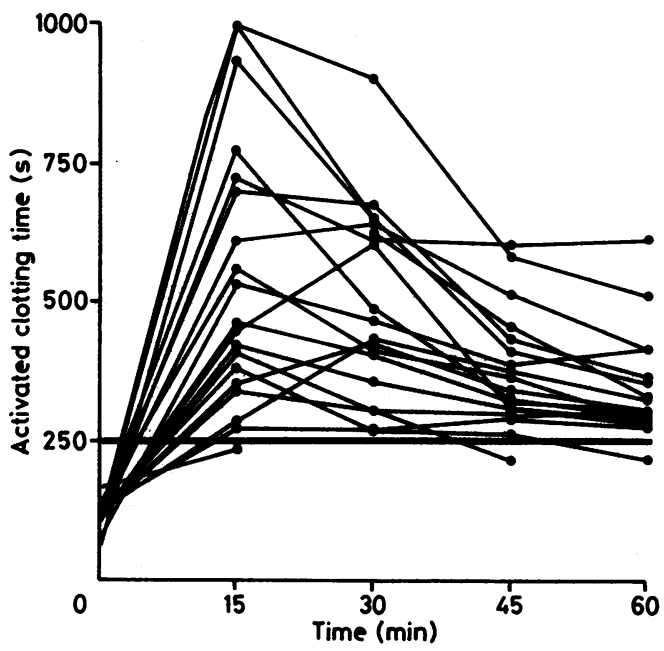

protocol II, three patients were inadequately anticoagulated at 15,45 , and 60 minutes. Thus $11(55 \%)$ patients in protocol I and three $(15 \%)$ patients in protocol II required additional doses of heparin.

Arteriovenous activated clotting time difference Figure 3 shows the mean arterial and venous activated clotting time values measured every 15 minutes in the 40 patients who were randomised to protocol I and II. In 28 patients $(70 \%)$ the arterial activated clotting time values were markedly higher during the first $30 \mathrm{~min}$ utes.

\section{Complications}

Several patients bled slightly around the sheath in the femoral artery during the 24 hour heparin infusion but did not require any therapeutic intervention. One patient bled profusely from around the arterial sheath during continuous heparin infusion and required blood transfusion. Her activated clotting time varied from 250 to $300 \mathrm{~s}$

\section{Factors affecting heparin activity}

Body weight, surface area, age, and sex did not affect the level of anticoagulation.

\section{Variability}

The variability in activated clotting time

Figure 3 Activated clotting time in arterial and venous blood.

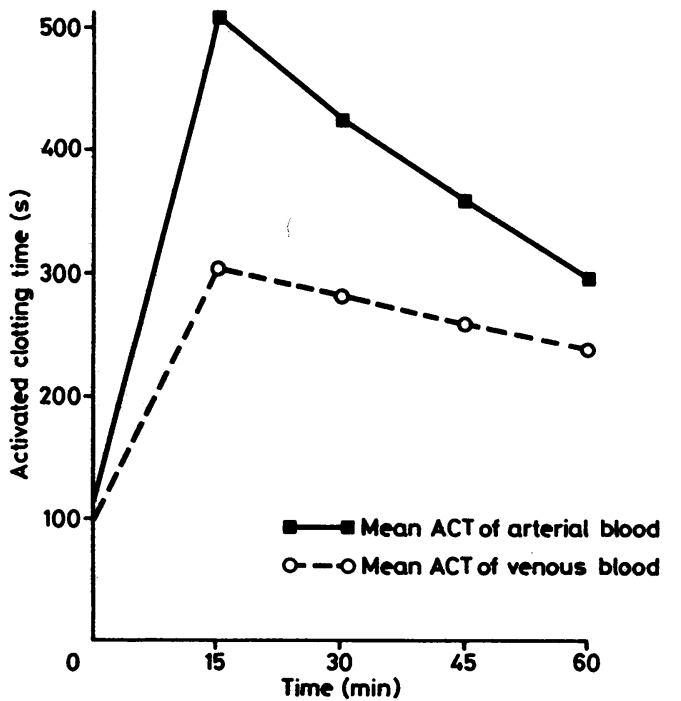

measurement was $<8 \%$ when two duplicate samples were analysed by a single operator and $<10 \%$ when they were analysed by two operators.

\section{Discussion}

Before we started the present study two of our patients had developed acute coronary occlusion during angioplasty of the left anterior descending artery and both had to undergo urgent coronary artery bypass surgery. Thrombi were found not only at the site of balloon inflation but also in the left circumflex artery. Both the patients had received 10000 units of heparin but were found to be inadequately anticoagulated (activated clotting time $<200 \mathrm{~s}$ ). This led us to monitor heparin induced systemic anticoagulation during angioplasty.

Heparin binds to circulating antithrombin III. The resulting complex inhibits thrombin and other coagulation factors. Heparin prevents thrombin from inducing platelet aggregation and is also believed to have a fibrinolytic action. ${ }^{9}$

The mean half life of heparin activity has been reported as being between 60 and 90 minutes. ${ }^{10} 11$ In 1975, Bull et al using manual measurement of activated clotting time reported that the individual sensitivity to heparin varied threefold and heparin half life varied fourfold. ${ }^{3}$ Our results confirm the wide variation in sensitivity to heparin and in the duration of effective anticoagulation. This may be because of individual differences in heparin clearance or variations in concentrations of circulating coagulation factors such as antithrombin III, platelet factor 4, and heparin neutralising proteins.

It is common practice to give 10000 units of heparin before percutaneous transluminal coronary angioplasty and a further 5000 units if the procedure extends beyond one hour. ${ }^{2}$ Heparin administered according to this protocol, however, will leave a significant number of patients inadequately anticoagulated, exposing them to the risk of thrombotic complications. One study has shown that $73 \%$ of coronary artery occlusions were related to ineffective anticoagulation. ${ }^{12}$. Hollman et al reported that $85 \%$ of acute occlusions occurred at the time of diminishing anticoagulation. ${ }^{2}$ Though both studies refer to acute occlusion after leaving the catheter laboratory, the results show the importance of adequate anticoagulation.

Our results show that to maintain effective anticoagulation during percutaneous transluminal coronary angioplasty, systemic anticoagulation induced by heparin should be monitored. There is controversy about the optimal in vitro test for monitoring heparin treatment. The consensus is that the degree of prolongation of anticoagulation effect gives a better assessment than heparin concentration, because the heparin effect reflects the balance between the direct action of the drug on clotting factors and the ability of other substances to potentiate or antagonise it. 
The tests that are commonly used are whole blood clotting time, activated clotting time, and activated partial thromboplastin time. Each test has its drawbacks. Whole blood clotting time is time consuming and there is lack of reproducible results. ${ }^{13}$ Activated partial thromboplastin time is a popular laboratory test and is considered to be sensitive but it is time consuming and cannot be easily performed in the catheterisation laboratory. Furthermore, its relation to the heparin concentration is linear only over a small heparin dosage range. ${ }^{14}$ The result also varies according to the reagent and instrument used. ${ }^{15}$

We found the automated activated clotting time (Haemochron and HemoTec) tests simple, inexpensive, and easy to use. They gave reproducible results. Tests performed on whole blood have two advantages. They can be performed quickly and they reflect the antiheparin activity of the platelets. Both produced results rapidly enough for the titration of heparin in the catheterisation laboratory or coronary care unit.

Bull et al recommended that the activated clotting time during cardiopulmonary bypass should be maintained at $>300 \mathrm{~s}$ to avoid clot formation. ${ }^{3}$ Young et al reported that activated clotting time should be maintained at $>400 \mathrm{~s}$ to prevent microemboli in the extracorporal circulation. ${ }^{16}$ Schriever et al found that the activated clotting time gave a strong linear correlation with blood heparin concentration unlike the activated partial thromboplastin time. They concluded that an activated clotting time value between 1.6 and 2.2 times baseline provides effective anticoagulation. ${ }^{17}$ We arbitrarily chose to maintain our patients' activated clotting time at $>250 \mathrm{~s}$, which was $>2.5$ times the baseline in most patients. We chose this value because we thought that patients undergoing percutaneous transluminal coronary angioplasty require less heparin than patients on cardiopulmonary bypass but they need sufficient heparin to prevent thromboembolism.

Heparin, in addition to preventing thrombus formation, inhibits platelet to platelet interaction and has also been shown experimentally to suppress the smooth cell proliferation, which contributes to the phenomenon of restenosis. ${ }^{18}$ We continued heparin infusion for 24 hours after percutaneous transluminal coronary angioplasty to assess its effect on restenosis. The results showed no significant reduction in the incidence of restenosis in the patients who were adequately anticoagulated compared with our previous 80 consecutive patients undergoing angioplasty or compared with reported rates of restenosis.

In the subsequent study we found that the activated clotting time values of arterial blood were considerably higher than those of venous blood. We do not think that intermittent flushing of the arterial line with saline containing a very small amount of heparin (approximately $30-40 \mathrm{ml}$ saline containing $<200$ units of heparin) explains the difference. The differences may be either related to heparin metabolism or possibly to the anticoagulant effect of the contrast media. Our present knowledge of heparin metabolism is insufficient and further studies are necessary to explain the discrepancy. We recommend activated clotting time monitoring of the arterial blood collected from the guiding catheter because this is more likely to reflect the level of anticoagulation at the site of angioplasty.

Heparin is obtained commercially from porcine intestinal mucosa or bovine lungs. The activities of these two preparations are identical. ${ }^{19} \mathrm{We}$ used heparin obtained from porcine intestinal mucosa.

We conclude that a bolus of 10000 units of heparin together with an infusion of 2000 units per hour resulted in adequate anticoagulation in most patients. Activated clotting time should be monitored in all patients to identify those who may be at higher risk of thromboembolic complications because of inadequate anticoagulation. Arterial values of activated clotting time are likely to be higher than venous measurements. A large study is necessary to validate the clinical effectiveness of adequate heparin anticoagulation.

1 Bettman MA. Anticoagulation and restenosis after percutaneous transluminal angioplasty. Am J Cardiol 1987; 60:17B-9B.

2 Hollman J, Gruentzig AR, Douglas JS Jr, King SB III, Ischinger T, Meier B. Acute occlusion after percutaneous transluminal angioplasty - a new approach. Circulation 1983;68:725-31.

3 Bull BS, Korpman RA, Huse WM, Briggs BD. Heparin therapy during extracorporal circulation: 1 . Problems inherent in existing heparin protocol. $J$ Thorac Cardiovasc inherent in existing hepar

4 Hattersley PG. The activated coagulation time of the whole blood (ACT). Progress report. Am J Clin Pathol 1976;66:899-903.

5 Chesebro JH, Lam JYT, Badimon L. Restenosis after arterial angioplasty: a haemorrheology response to injury. Am J Cardiol 1987;60:10B-6B.

6 Bredlau CE, Gary SR, Leimgruber PP, Doughlas JS Jr, King SB III, Gruentzig AR. In-hospital morbidity and mortality in patients undergoing elective coronary angioplasty. Circulation 1985;72:1044-52.

7 Park JH, Bettman MA, Adelman B, et al. In vivo imaging and evaluation of platelet accumulation vs. time at arterial injury site. Invest Radiol 1985;20:287-92.

8 Steele PM, Chesebro JH, Stanson AW, et al. Balloon angioplasty. Natural history of the pathophysiological response to injury in a pig model. Circ Res 1985;57: 105-12.

9 Markwardt F, Klocking HP. Heparin induced release of plasminogen activator. Haemostasis 1977;6:370-4.

10 Olssen $P$, Lagingren $H, E k S$. The elimination from plasma of intravenous heparin. An experimental study on dogs and humans. Acta Med Scand 1963;173:619-30.

11 Estes JW. Kinetics of anticoagulant effect of heparin. JAMA 1970;212:1492-5.

12 Gabliani G, Deligonul U, Kern MJ, Vandormael M. Acute coronary occlusion occurring after successful transluminal coronary angioplasty: temporal relationship to discontinuation of anticoagulation. Am Heart J discontinuation of

13 Hill J, Dontigny L, De Leval M, Mielke CH Jr. A simple method of heparin management during prolonged extracorporal circulation Ann Thorac Surg 1974;17 129-34.

14 Koepke JA, Triplett DA, Bunez G. The partial thromboplastin time for monitoring heparin therapy. In: Lundblock R, ed. Chemistry and biology of heparin. New York Elsevier North-Holland, 1981:625.

15 Triplett DA, Horms CA, Koepke JA. The effect of heparin on the activated partial thromboplastin time. Am J Clin Pathol 1978;70:556-9.

16 Young JA, Kisher CT, Doty DB. Adequate anticoagulation during cardiopulmonary bypass determined by activated clotting time and the appearance of fibrin monomer. Ann Thorac Surg 1976;26:231-4.

17 Schriever HG, Epstein SF, Mintz MD. Statistical correlation and heparin sensitivity of activated partial thromboplastin time, whole blood coagulation time and automated coagulation time. Am J Clin Pathol 1973;60:323-9.

18 Clowes AW, Clowes MM. Kinetics of cellular proliferation after arterial injury. II. Inhibition of smooth muscle growth by heparin. Lab Invest 1985;52:611-6.

19 Jain AK, McMahon FG, Ryan JR. Comparison of anticoagulant activity of three preparations of heparin. Curr Ther Res 1977;22:427-37. 